\title{
Schistosomiasis and soil-transmitted helminth infections in schoolchildren in north-eastern Democratic Republic of the Congo
}

\author{
Astrid M. Knoblauch ${ }^{a, b, *}$, Colleen Archer $^{c}$, Milka Owuor ${ }^{d}$, Sandro Schmidlin ${ }^{a, b}$, Mark J. Divall ${ }^{d}$, \\ Jürg Utzinger ${ }^{a, b}$ and Mirko S. Winkler ${ }^{a, b}$ \\ ${ }^{a}$ Swiss Tropical and Public Health Institute, P.O. Box, CH-4002 Basel, Switzerland; 'University of Basel, P.O. Box, CH-4003 Basel, \\ Switzerland; 'University of KwaZulu-Natal, 4041 Durban, South Africa; ${ }^{d}$ SHAPE Consulting Limited, P.O. Box 602, St Peter Port, \\ Channel Islands \\ *Corresponding author: Tel: +41 61 284-8713; Fax: +41 61 284-8105; E-mail: astrid.knoblauch@unibas.ch
}

Received 17 March 2016; revised 30 May 2016; accepted 31 May 2016

Background: There is a paucity of epidemiological data pertaining to schistosomiasis and soil-transmitted helminth (STH) infections in the Democratic Republic of the Congo (DRC).

\begin{abstract}
Methods: A cross-sectional survey was carried out in the north-eastern part of DRC enrolling 400 schoolchildren aged 9-14 years. Stool and urine samples were subjected to standard diagnostic methods and examined under a microscope for helminth eggs.
\end{abstract}

Results: Four out of five children were infected with at least one helminth species. Schistosoma mansoni was the predominant species (57.8\%). Urine examinations were all negative for S. haematobium.

Conclusions: S. mansoni and STH infections are highly endemic in the surveyed part of the DRC, calling for interventions in school-aged children.

Keywords: Cross-sectional survey, Democratic Republic of the Congo, Schistosomiasis, Soil-transmitted helminthiasis

\section{Introduction}

The Democratic Republic of the Congo (DRC) is considered a high-burden country for schistosomiasis and soil-transmitted helminthiasis, yet this claim is based on very scattered data. ${ }^{1-4}$ We present findings from a cross-sectional survey carried out in nine villages in the Watsa health zone in the north-eastern part of DRC. School-aged children were subjected to standard, quality-controlled diagnostic methods and results were fed into the open-access Global Neglected Tropical Diseases database. ${ }^{5}$

\section{Materials and methods}

The study area is situated in north-eastern DRC in the Watsa health zone in Haut-Uélé province, approximately $190 \mathrm{~km}$ north-west of the town of Bunia. A cross-sectional survey was carried out in August 2010, during the rainy season, in primary schools in nine villages around the settlement of Durba (see Supplementary Figure 1). In each school, a minimum of 30 children aged 9-14 years were randomly selected. In total, 400 children were sampled, comprising 213 boys (53.3\%) and 187 girls.
From each child, a fresh morning stool sample and a midday, post-exercise urine sample were collected. The samples were processed using standard, quality-controlled methods. For stool samples, a single $41.7 \mathrm{mg}$ Kato-Katz thick smear was prepared on slides and analysed under a microscope. ${ }^{6}$ The number of eggs of Schistosoma mansoni, Ascaris lumbricoides, hookworm, and Trichuris trichiura were counted and multiplied by a factor of 24 to obtain an infection intensity measure; i.e., eggs per gram of stool (EPG). ${ }^{6}$ Urine samples were examined for S. haematobium, using a $10 \mathrm{ml}$ centrifugation method. ${ }^{7}$

Statistical analyses were performed with STATA, version 13 (StataCorp., College Station, TX, USA). Descriptive statistics for frequency, including 95\% confidence intervals, were prepared. Geometric mean intensity of helminth infection was used in the analysis. The $p$-values were calculated using Pearson's $\chi^{2}$ test of proportions.

\section{Ethical approval}

The purpose and procedures of the study were explained to village leaders, school teachers, and schoolchildren. Written informed consent was obtained from school directors and 
teachers, while children assented orally. At the end of the survey, all children received albendazole (single dose, $400 \mathrm{mg}$ ) against soil-transmitted helminthiasis, whilst children with a confirmed S. mansoni infection were treated with praziquantel (single dose, $40 \mathrm{mg} / \mathrm{kg}$ ).

\section{Results}

S. mansoni was the predominant helminth species; 231 children were found with egg-positive Kato-Katz thick smears, thus resulting in a prevalence estimate of $57.8 \%$ (Table 1). The prevalence increased with age (e.g., children aged 9-10 years had a prevalence of $48.2 \%$, while children in the oldest age group, 13-14 years, had a prevalence of $69.9 \% ; p=0.001$ ). No S. haematobium eggs were found in urine samples subjected to a filtration method.

Hookworm was the most common soil-transmitted helminth (STH) species (prevalence: $48.5 \%$ ). Boys had a significantly higher hookworm prevalence than girls $(55.4 \%$ vs $40.6 \%, p=0.003)$. T. trichiura was found at a relatively low level $(5.0 \%)$, while no A. lumbricoides were found.

Among the S. mansoni-infected children, more than half $(52.8 \%)$ were heavily infected ( $\geq 400 \mathrm{EPG})$, whilst an additional $28.6 \%$ had a moderate infection intensity (100-399 EPG). For hookworm, most infections were of light intensity with fewer than 2000 EPG (85.1\%).

Overall, 140 children (35.0\%) were infected with at least two species of intestinal helminths (data not shown). S. mansonihookworm co-infection affected more than a quarter of the study participants (25.5\%). Nine children (2.3\%) were found with a triple species infection (i.e., S. mansoni, hookworm and T. trichiura)

\section{Discussion}

We found high prevalence and intensity of S. mansoni infection among 9- to 14-year-old school-going children (overall prevalence was $57.8 \%$ ) during a cross-sectional survey conducted in August 2010 in the north-eastern part of DRC. Compared with previous studies, our findings suggest that this part of northeastern DRC is more highly endemic for $S$. mansoni than expected. ${ }^{8,9}$ Discrepancies between modelled and surveyed estimates for S. mansoni were also found in highly urbanised settings of DRC. ${ }^{4,9}$ Furthermore, we did not find a single S. haematobium infection, which is in contrast to previous estimates made by WHO that suggested that between 10 and $50 \%$ of children in this province are infected with S. haematobium. ${ }^{9}$

The infection prevalence for T. trichiura (5.0\%) and A. lumbricoides $(0.0 \%)$ were considerably lower than previously modelled rates. ${ }^{3,10}$ The hookworm prevalence ( $48.5 \%$ in this study) was found within the range of previous predictions by KaragiannisVoules et al. who modelled a rate above $50 \% .^{3}$

Although DRC is one of three high-burden countries in Africa for helminthiases and other neglected tropical diseases, the country has yet to set-up a national control programme for neglected tropical diseases. ${ }^{2,8}$ The lack thereof was underscored by local health and education authorities reporting that none of the schools had ever received preventive chemotherapy against schistosomiasis and soil-transmitted helminthiasis.

Table 1. Prevalence of Schistosoma mansoni and soil-transmitted helminth infection in the Durba study area, Democratic Republic of the Congo, 2010

\begin{tabular}{|c|c|c|c|c|c|}
\hline & $\mathrm{n}$ & Schistosoma mansoni & Hookworm & Trichuris trichiura & Any helminth \\
\hline \multicolumn{6}{|c|}{ Prevalence of infection, $\%$ (95\% CI) } \\
\hline \multicolumn{6}{|l|}{ Sex } \\
\hline Male & 213 & $60.1(53.5-66.7)$ & $55.4(48.7-62.1)$ & $6.6(3.2-9.9)$ & $83.1(77.4-87.9)$ \\
\hline Female & 187 & $55.1(47.9-62.2)$ & $40.6(33.5-47.7)$ & $3.2(0.6-5.8)$ & $78.1(71.5-83.8)$ \\
\hline \multicolumn{6}{|l|}{ Age (years) } \\
\hline $9-10$ & 193 & $48.2(41.1-55.3)$ & $36.8(29.9-43.7)$ & $2.1(0.0-4.1)$ & $72.0(65.1-78.2)$ \\
\hline $11-12$ & 114 & $64.0(55.1-73.0)$ & $57.0(47.8-66.2)$ & $7.9(2.9-12.9)$ & $87.7(80.3-93.1)$ \\
\hline $13-14$ & 93 & $69.9(60.4-79.4)$ & $62.4(52.3-72.4)$ & $7.5(2.1-13.0)$ & $90.3(82.4-95.5)$ \\
\hline Total & 400 & $57.8(52.9-62.6)$ & $48.5(43.6-53.4)$ & $5.0(2.9-7.1)$ & $80.1(76.5-84.5)$ \\
\hline \multicolumn{6}{|c|}{ Geometric mean EPG, n (95\% CI) } \\
\hline Total & 400 & $397.8(327.9-482.6)$ & $456.1(366.3-567.9)$ & $118.5(73.4-191.1)$ & NA \\
\hline \multicolumn{6}{|c|}{ Intensity of infection, \% ( $95 \%$ CI) } \\
\hline Infected, $\mathrm{n}$ & & 231 & 194 & 20 & NA \\
\hline Light $^{\mathrm{a}}$ & & $18.6(13.8-24.2)$ & $85.1(79.2-89.8)$ & $100.0(83.1-100.0)$ & NA \\
\hline Moderate $e^{b}$ & & $28.6(22.8-34.9)$ & $6.2(3.2-10.6)$ & $0.0(0.0-0.0)$ & NA \\
\hline High $^{c}$ & & $52.8(46.2-59.4)$ & $8.8(5.2-13.7)$ & $0.0(0.0-0.0)$ & NA \\
\hline
\end{tabular}


Our study has limitations that are offered for consideration. First, the data presented here were obtained from a crosssectional survey in selected villages, and hence, cannot be generalised for a wider area as infection with Schistosoma spp. and STHs show focal distribution. Second, the true prevalence rates are likely to be higher due to the low sensitivity of single KatoKatz thick smears. ${ }^{11}$

\section{Conclusions}

Our findings from an undersurveyed area suggest that helminth infections are widespread in north-eastern DRC and that polyparasitism is common. Previous, modelled estimates for the study area did not fully corroborate with our results. The data have been georeferenced and fed into the open-access Global Neglected Tropical Diseases database for subsequent risk profiling, which will help shape spatial targeting of control interventions. ${ }^{5}$

\section{Supplementary data}

Supplementary data are available at Transactions online (http://trstmh.oxfordjournals.org/).

Authors' contributions: CA, SS, MJD and MSW conceived the study and designed the study protocol; AMK, CA, MO, MJD and MSW carried out the field study; AMK, CA and MSW analysed and interpreted data. AMK and MSW drafted the manuscript; AMK, MJD, JU and MSW critically revised the manuscript for intellectual content. All authors read and approved the final manuscript. AMK and MSW are the guarantors of the paper.

Acknowledgements: Thanks are addressed to the health district teams of Haut-Uélé East and Bunia and the Laboratoire Médical de Référence de l'Ituri for their support and technical assistance during the field work and laboratory investigations. Special thanks are addressed to the education authorities, including the indispensable support and collaboration of the teaching staff. The authors are grateful to the children and parents for their willingness to participate in the study.

Funding: This work was supported by Randgold Resources. The funder had no role in the data collection, analysis, decision to publish, or preparation of the manuscript. No specific funding has been received for writing up this manuscript.
Competing interests: None declared.

Ethical approval: The study was approved by the health district of HautUélé East, Orientale province, Ministry of Health (reference no. 771/047/ BDS/HU-E/WAT/2010).

\section{References}

1 Rimoin AW, Hotez PJ. NTDs in the heart of darkness: the Democratic Republic of Congo's unknown burden of neglected tropical diseases. PLoS Negl Trop Dis 2013;7:e2118.

2 Hotez PJ, Kamath A. Neglected tropical diseases in sub-Saharan Africa: review of their prevalence, distribution, and disease burden. PLoS Negl Trop Dis 2009;3:e412.

3 Karagiannis-Voules DA, Biedermann P, Ekpo UF et al. Spatial and temporal distribution of soil-transmitted helminth infection in subSaharan Africa: a systematic review and geostatistical metaanalysis. Lancet Infect Dis 2015;15:74-84.

4 Matangila JR, Doua JY, Linsuke S et al. Malaria, schistosomiasis and soil-transmitted helminth burden and their correlation with anemia in children attending primary schools in Kinshasa, Democratic Republic of Congo. PLoS One 2014;9:e110789. doi:10.1371/journal. pone.0110789.

5 Hürlimann E, Schur N, Boutsika K et al. Toward an open-access global database for mapping, control, and surveillance of neglected tropical diseases. PLoS Negl Trop Dis 2011;5:e1404.

6 Katz N, Chaves A, Pellegrino J. A simple device for quantitative stool thick-smear technique in schistosomiasis mansoni. Rev Inst Med Trop São Paulo 1972;14:397-400.

7 Hodges M, Koroma M, Baldé MS et al. Current status of schistosomiasis and soil-transmitted helminthiasis in Beyla and Macenta prefecture, Forest Guinea. Trans R Soc Trop Med Hyg 2011;105:672-4.

8 Rollinson D, Knopp S, Levitz S et al. Time to set the agenda for schistosomiasis elimination. Acta Trop 2013;128:423-40.

9 WHO. Preventive chemotherapy and transmission control - country profile: Democratic Republic of the Congo. Geneva: World Health Organization; 2010.

10 Brooker S, Clements ACA, Bundy DAP. Global epidemiology, ecology and control of soil-transmitted helminth infections. Adv Parasitol 2006;62:221-61.

11 Glinz D, Silué KD, Knopp S et al. Comparing diagnostic accuracy of Kato-Katz, Koga agar plate, ether-concentration, and FLOTAC for Schistosoma mansoni and soil-transmitted helminths. PLoS Negl Trop Dis 2010;4:e754. 